\title{
Controller Design of Power Quality-Improving Appliances
}

\author{
D. J. Hammerstrom, Senior Member, IEEE, N. Zhou, Member, IEEE, and N. Lu, Senior Member, IEEE \\ Pacific Northwest National Laboratory \\ 902 Battelle Blvd., P. O. Box 999 \\ Richland, WA 99352 USA
}

\begin{abstract}
This paper presents an innovative solution to power quality problems using power quality-improving (PQI) appliances. PQI appliances conduct currents that supplement and correct the sum of the other load currents within a premise. From the utility side, the premise housing a PQI appliance thus becomes an improved, if not ideal, utility customer. The PQI appliance improves both harmonic power quality and power factor while performing its normal function, such as heating water. In this paper, the water heater PQI appliance is used as an example to demonstrate the control circuit design and function. Both computer simulation results and laboratory experiment results are presented to demonstrate the effectiveness of the approach. The estimated costs of the PQI controller and of harmonic compensating filters are compared to show that the PQI appliance may be an economic way to provide power quality improvement at the building level.
\end{abstract}

\section{INTRODUCTION}

The proliferation of nonlinear loads, such as static power converters, has deteriorated power quality in power distribution systems [1]. Consumer electronics and battery chargers drawing harmonic currents result in excess heat losses and low power factors in electrical distribution and transmission equipment. Poor power factors contribute to voltage instabilities and incur losses in distribution lines. Power factor eventually must be corrected by capacitor banks or by synchronous condensers. In addition, harmonics can excite system modes and cause a distribution system to resonate at a critical frequency. Distorted current waveforms can also lead to distribution voltage distortion in cases where system impedances are high. Thus, an electric customer who operates poor power quality loads can affect his neighbor's electrical power quality, too. Other negative impacts of harmonics include EMI and RFI noises and interferences with communication systems [2], [3].

To mitigate, cancel, block, or reduce the unwanted harmonic contents, filtering techniques are commonly used. Commercially available harmonic filter equipment falls into three categories: active filters, passive filters, and hybrid filters [4]. A common characteristic of harmonic filters is that large energy storage devices such as capacitors, inductors, or batteries must be used to compensate, isolate or mitigate the harmonics. A filter must absorb energy from one part of the electrical waveform, store that energy, and re-inject it

This work is supported by the Pacific Northwest National Laboratory, operated for the U.S. Department of Energy by Battelle under Contract DEAC06-76RL01830. elsewhere to perform its function. Harmonic filters are generally expensive and result in additional energy losses [5]. Power factor may be corrected for individual electrical loads that possess rectifying power supplies [7], [8], but this practice is not universally applied.

In this paper, we introduce a type of appliance and controller that can accomplish the functions of traditional filters by selectively consuming, rather than absorbing and reinjecting, the energies of unwanted harmonics and imperfect power factor. We introduce the name "power qualityimproving appliances” (PQI) to describe these devices. PQI appliances monitor the current and voltage waveforms at the service panel of a building and shape the current they themselves consume so that the sum of electrical currents consumed by the entire building exhibit improved, if not perfected, harmonic power quality and unity power factor. PQI appliances may cost less than traditional filters, and they may provide a more electrically efficient way to mitigate harmonics.

The paper is organized as follows. Section II describes the control method and objective. Section III describes the circuit configurations of the PQI controller. Section IV describes the SIMULINK simulation results. Experimental setups and results are discussed and modeled in Section V. Section VI provides conclusions.

\section{CONTROL SCHEME AND OBJECTIVES}

We first present some figures that will clarify the control being implemented for harmonic and power factor improvements using PQI appliances.

Starting from the bottom and progressing upward, Fig. 1 shows the following four waveforms: (1) The bottom is an electrical cycle of load current that exhibits two deep notches. (2) The second waveform is the resulting PQI appliance current where the PQI appliance responds to the notches but controls a small load or has low gain setting. (3) The next waveform is a PQI appliance current that is ideally matched to the power quality challenge, such that the sum of the PQI and other premise currents will be a completed sinusoid. (4) The topmost waveform is a PQI appliance current that not only supplements the currents in the notches, but also adds everywhere to the sinusoidal current envelope. These top three waveforms of Fig. 1 would accompany increasing PQI appliance controller gain from bottom to top. 


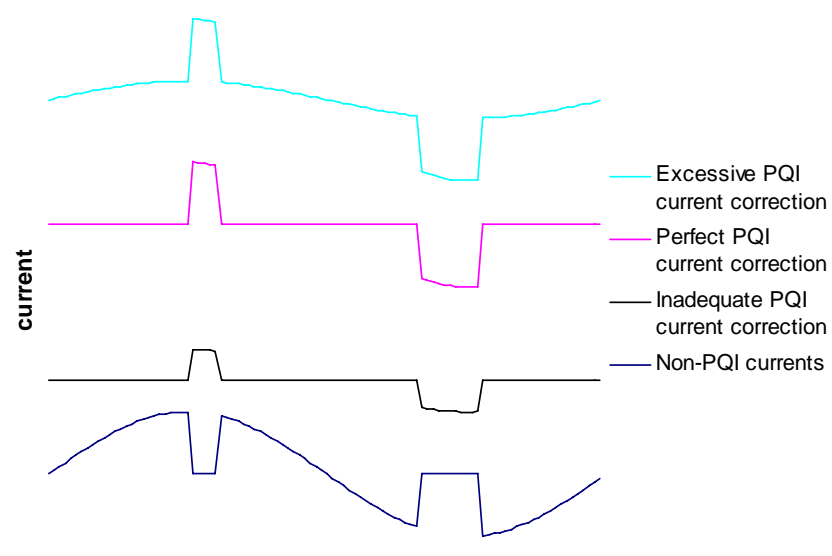

time

Figure 1. Three PQI appliance current waveforms showing the effect of PQI appliance gain setting for the correction of a current waveform having two deep notches.

In effect, the PQI controller monitors the entire premise's current and attempts to force the current to become a scaled version of the voltage signal at the premise. Those familiar with power factor correction chips will recognize this strategy that is commonly used for power factor correction input stages on rectifiers. In fact, the recognition of this similarity permitted us to simplify our PQI controller's design by adopting one of the many available inexpensive power factor control chips.

We saw in Fig. 1 that the setting of a gain and the relative magnitude of the PQI appliance load can affect the degree to which power quality can be improved. Furthermore, a PQI appliance is limited to only the consumption of current and, unlike a filter, must always conduct in the direction dictated by the sign of the voltage waveform.

Fig. 2 demonstrates the limitation even better. Fig. 2 shows how a PQI appliance attempts to improve a premise's current waveform, the phase of which has been significantly shifted by reactive loads at the premise. The PQI appliance current must always have the same sign as the applied voltage. At the left of Fig. 2(b), the PQI appliance current begins to follow a sinusoidal trajectory as it corrects the negative premise current. The PQI appliance current becomes zero when the error signal difference between the PQI sinusoidal current envelope and actual system currents would intersect. The PQI appliance current then remains zero for the rest of the half cycle because current conduction in the PQI appliance cannot be reversed during any voltage half cycle.

Fig. 2(a) shows the sum of the PQI appliance current and the other premise currents. While the current waveform has not been perfected, it has been improved. Energy has been put to good use (heating water, for example), and the premise's phase shift has been reduced. This example resulted in an interesting tradeoff between the premise's current phase shift and reduced harmonic power quality.

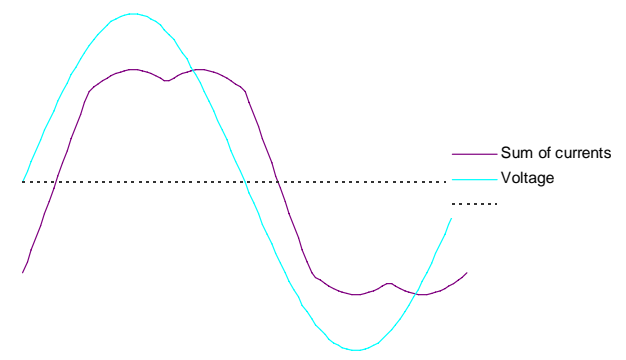

(a)

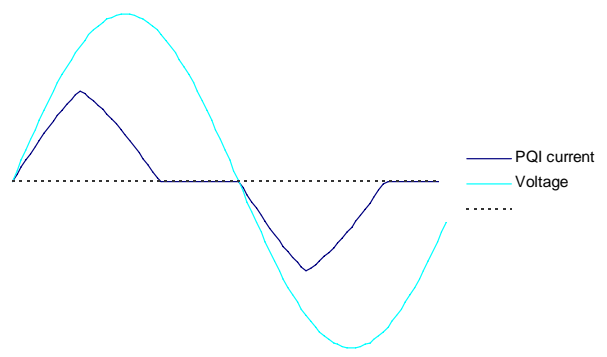

(b)

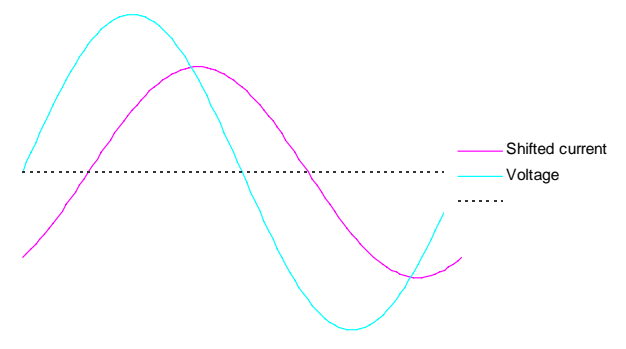

(c)

Figure 2. (a) Sum of the PQI appliance current and remaining premise loads, (b) the PQI appliance current by itself, and (c) the premise voltage and phaseshifted current waveforms.

\section{SYSTEM CIRCUIT CONFIGURATIONS}

A PQI appliance system requires three components: voltage and current metering at the electrical service entrance, the controller, and the appliance load. Refer to Fig. 3.

Instantaneous voltage and current measurements must be obtained at the electrical service panel. The measurements must be point-on-wave, not RMS, to facilitate fast corrections within each electrical cycle. Ideally, bandwidth attenuation of the voltage and frequency measurements will occur above 1 $\mathrm{kHz}$, or so, but below the PQI appliance's PWM frequency.

The communication of these signals from the electrical service panel to the PQI controller admittedly presents a technical challenge that was not resolved during the present research, development, or laboratory testing. We propose, however, that this technical obstacle may be overcome by a means of wireless communication.

The function of the PQI controller block of Fig. 3 is to accept the voltage and current measurements from the service panel and calculate a PWM output signal to control the current 


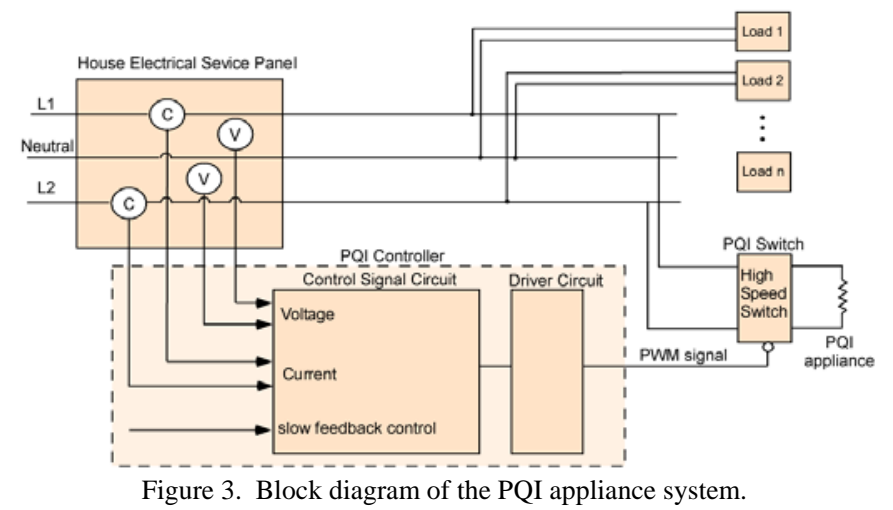

consumed by the PQI appliance. The input called "slow feedback control" is the controller's gain input. We foresee this gain input eventually being responsive to externally produced signals, including thermostat set points, power quality measurements, or demand response signals, for example.

While shown as part of the PQI controller, the driver circuits should reside very near the PQI appliance and its power electronic switches.

Controller design can be greatly simplified by recognizing the similarities between our objectives and those of power factor controller packages. This similarity is made clear in Fig. 4. Power factor controllers possess a fast control loop and a slow one. In the fast loop, the voltage signal is multiplied with the slow feedback signal and the result is compared to the instantaneous current. This comparison determines the duty cycle of the next PWM signal.

Scaled, rectified representations of voltage and current are needed by the power factor controller. In boost converters, to which power factor controllers are often applied, rectification is done in hardware, and the scaled, rectified signals are easily obtained from simple resistor voltage dividers and across shunt resistors. Such was not the case for the PQI appliance. We chose instead to scale the voltage signal with a signal transformer, and we scaled the current with a current transformer. These input signals were then rectified within the PQI controller using precision electronic rectifiers.

Among many considered power electronic switch configurations, the chosen topology consists of two fast IGBT power switches identically referenced to the two ac voltage inputs of the appliances, as shown in Fig. 5. The dissipative

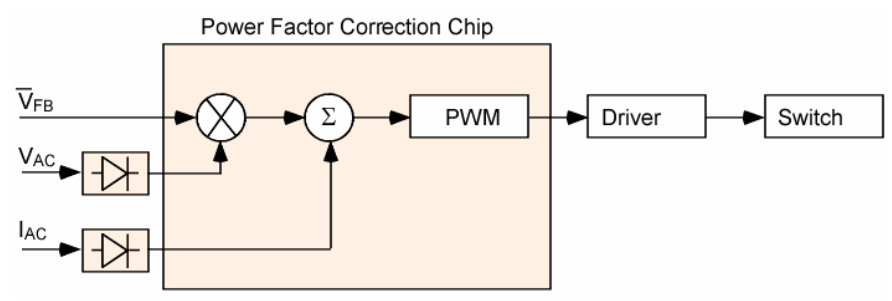

Figure 4. A commercially-available power factor controller can be used to simplify PQI appliance controller

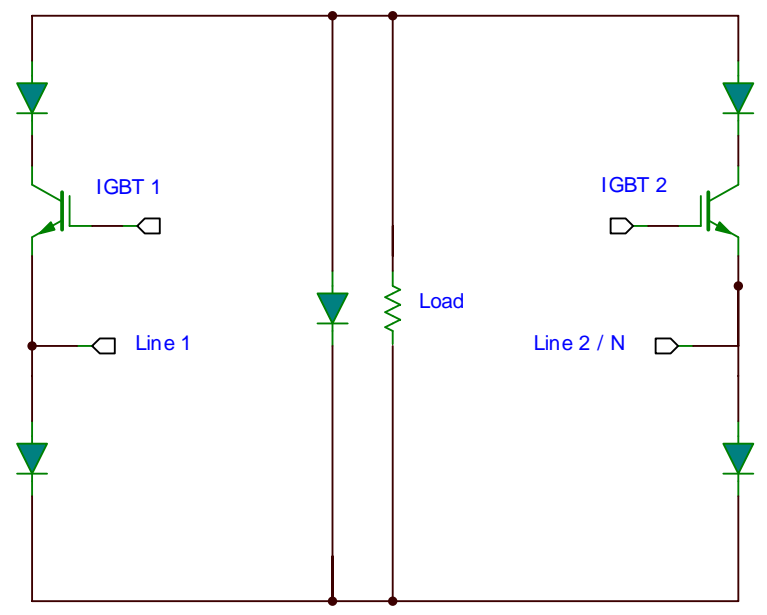

Figure 5. Power circuit of the PQI appliance.

load is shown at the diagram's center. This power circuit consists of two power electronic switches and five power diodes. Four of the diodes back up the power switches; the fifth wheels load current during switching transients and when the PQI load is reactive.

The major advantages of this topology are as follows: (1) the drive circuits may be easily powered from the ac inputs; (2) only two power switches are used, (3) identical PWM modulation signals may be sent to both switches; and (4) the wheeling diode allows inductive elements to be present in the appliance. Most important, any failure of any one electronic switch will result in no greater current than the appliance current, which is limited by the appliance impedance.

One of two driver circuits is shown in Figure 6. This clever design is possible because of the way the power switches have been referenced to the two line voltages. Starting at the left of the circuit, the floating driver power supplies originates from a center-tapped transformer. The center tap of the transformer for each driver circuit is tied to whichever line voltage is used as the reference by the driver. The center tap centers the driver circuit rails about the reference voltage. Both the high and low rail voltages are then carefully regulated to provide the large positive-going voltage and the smaller negative offbias voltage. The high-current MOSFET driver is turned on and off optically to drive the power switch gates.

\section{Simulation RESUlts}

Simulations were conducted for individual block diagram components in SPICE as well as the overall system in MATLAB $^{\mathrm{TM}}$. Fig. 7 demonstrates a noteworthy limitation of the PQI appliance: the PQI appliance can only remediate power factor and harmonic quality within the limitations imposed by an ac current envelope, which is the conduction that would occur through the appliance at $100 \%$ duty cycle. In this figure, the improvement is significant, but is insufficient 


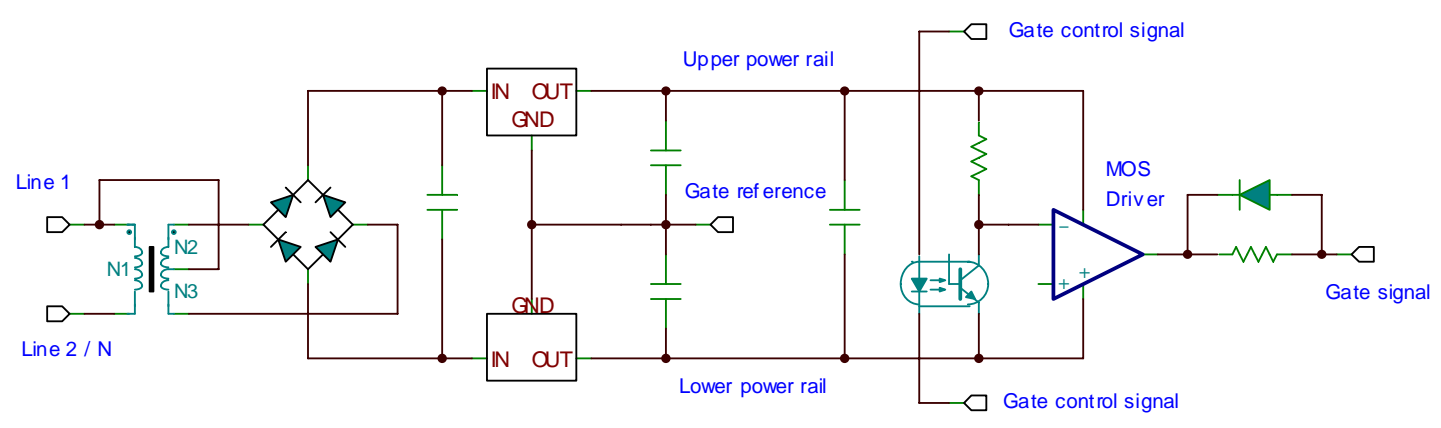

Figure 6. One of two PQI driver circuits.

to completely eliminate the large $300-\mathrm{Hz}$ current components and the extensive current lag.

\section{EXPERIMENTAL SETUPS AND RESUltS}

As shown in Fig. 8(a), a laboratory test bed was constructed, which permits us to vary the magnitude of the PQI appliance impedance and to submit the test appliance to a variety of power quality challenges. The test appliance scale is similar to a water heater $-5 \mathrm{~kW}$. We remain optimistic about the use of PQI water heaters because water heater loads normally constitute $10 \%$ - $20 \%$ of household load [6]. They could, on average, provide sufficient resources to mitigate the harmonic currents generated by households and most buildings.

The prototype PQI switch board, its power switch drivers and driver power supplies are shown in Fig. 8(b). The cost of this prototype PQI controller and hardware was about \$50 US, which is cheaper than some stand-alone power factor correctors and all active harmonic filters of similar magnitude. Only small heat sinks were required. Because a water heater is used as the target PQI appliance, an additional advantage is that even the heat put into the heat sinks could be put to use to heat water, making the theoretical efficiency of the PQI appliance approach unity.

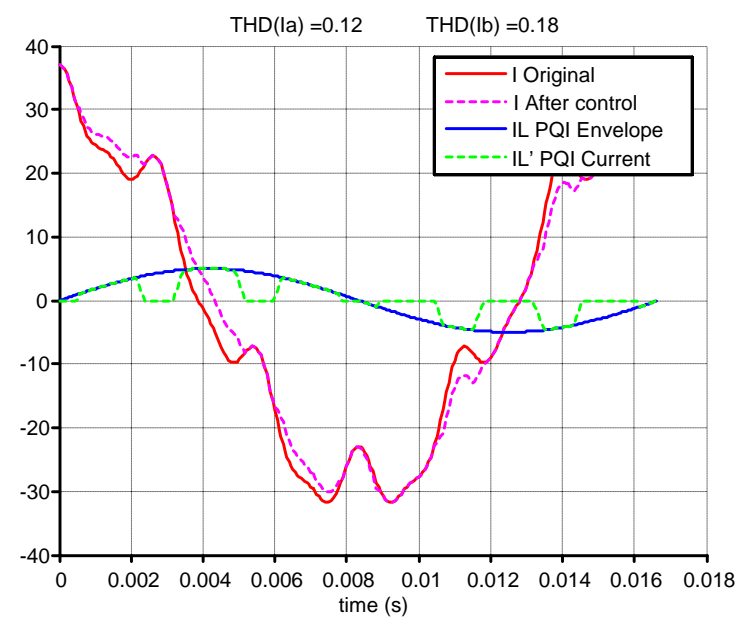

Figure 7. Matlab simulation result showing PQI appliance reaction to a 300$\mathrm{Hz}$ polluting current signal. The local loads also exhibit significant reactive phase shift.
The laboratory PQI device was designed to use Linear Technology's LT1249 power factor controller which operates at $100 \mathrm{kHz}$. An active feedback loop was designed around this controller's voltage amplifier to create a steady gain input with which the PQI can be made more or less responsive. The load bank of Fig. 8(a) can be stepwise adjusted to affect the impedance into which the PQI appliance switches and dissipates energy. The remainder of the premise load was emulated with an NHR model 4600 programmable ac load. This electronic load permitted us to independently control the premise current magnitude and power factor. All tests were performed with 120-Vac power input. Performance of the circuit was observed and recorded on a digital oscilloscope.

For now, we will be satisfied to observe and understand the qualitative behaviors of the PQI appliance as we modify several independent variables - PQI load, premise load, and PQI controller gain. We hope to follow up this research soon with more quantitative results.

The first series of data presented, Fig. 9, shows the effect of increasing power quality pollution from other premise loads. The PQI gain and impedance were set at intermediate values. The power factor of the 5- $\mathrm{A}_{\mathrm{RMS}}$ NHR electronic load was then changed from unity to 0.9 , to 0.8 , and so on. Representative oscilloscope current plots are shown in Fig. 9.

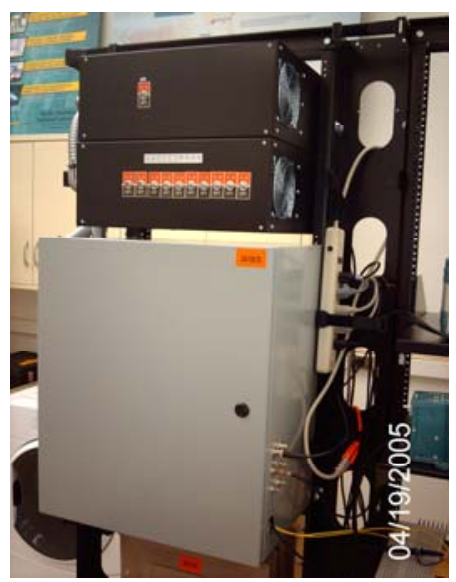

(a)

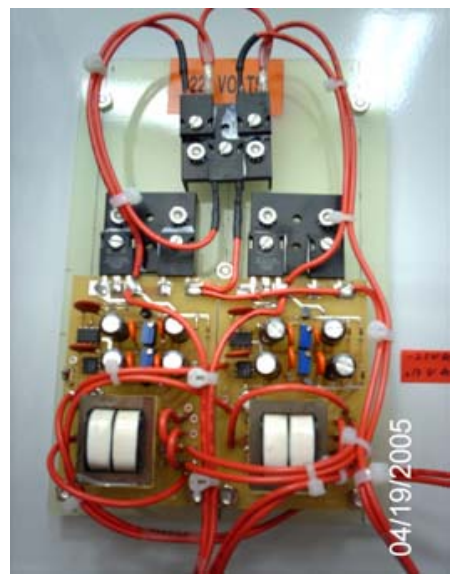

(b)
Figure 8. (a) Laboratory PQI test bed and (b) 5-kW PQI driver boards and power section. 


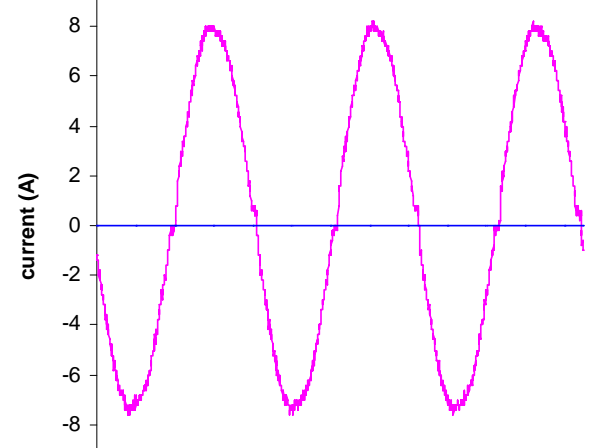

(a)

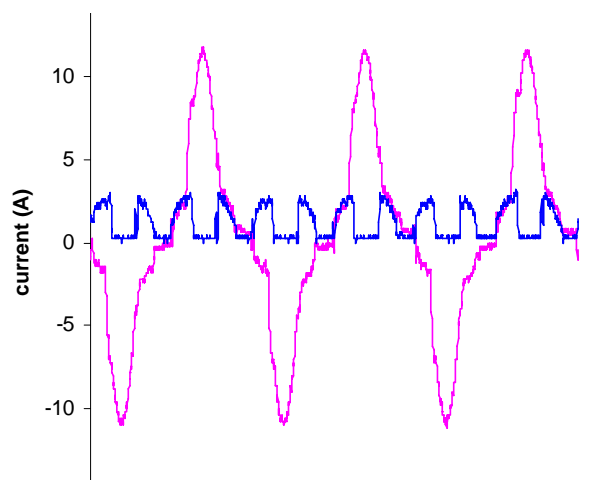

(b)

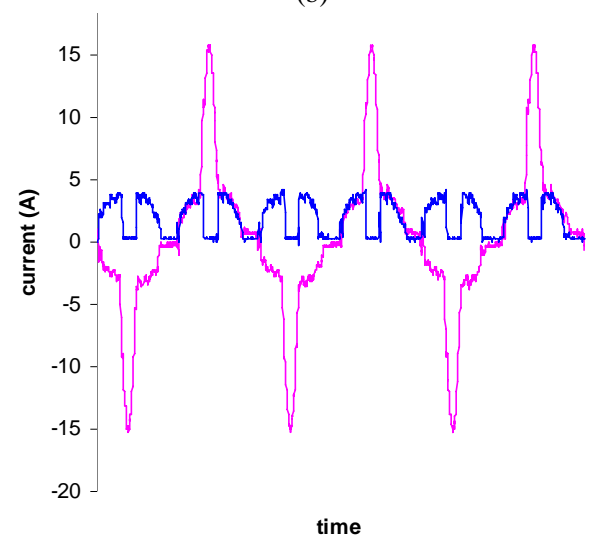

(c)

Figure 9. Laboratory PQI appliance responses to power factors (a) 1.0, (b) 0.8 , and (c) 0.6. Red line is applied 5-Arms load, and the blue line is the PQI current conduction response.

The next series of oscilloscope plots, Fig. 10, shows that the PQI appliance's current increases as its controller gain is increased. Observe that the PQI current is rectified. Rectification of the consumed current is a ramification of the power circuit design shown earlier in Fig. 5.

The final Fig. 11 demonstrates the effect of PQI appliance load size on its behavior. The magnitude of the emulated premise load is $5 A_{R M S}$, and the power factor of the premise load was held at 0.8. The PQI load in Fig. 11(a) is small and has a limited ability to correct the premise current. The larger PQI appliance load of Fig. 11(b) does a better job correcting the nonsinusoidal premise current.

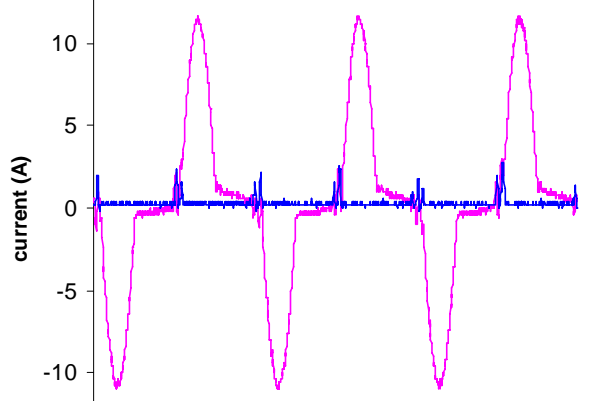

(a)

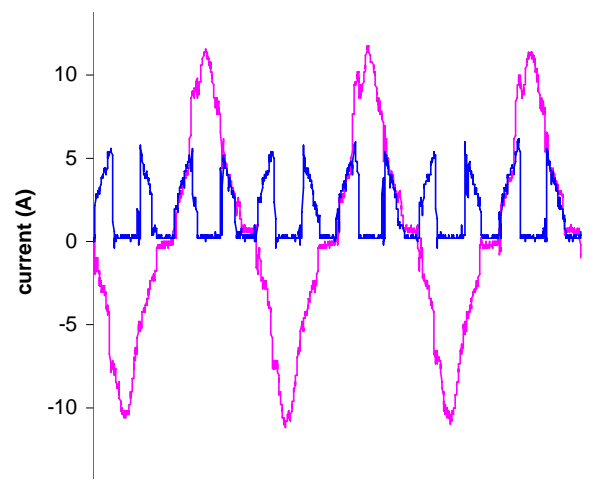

(b)

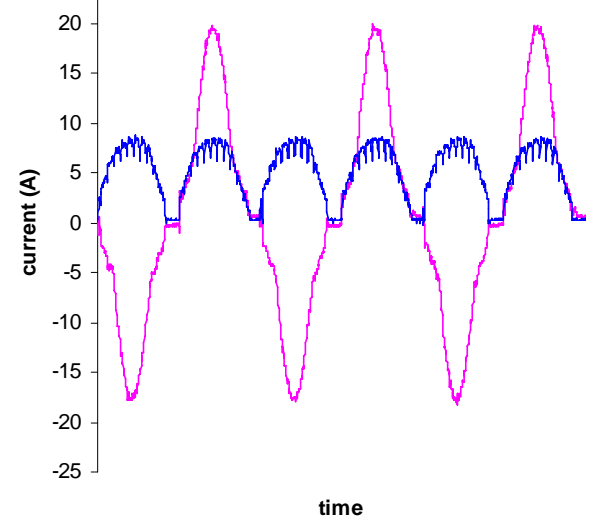

(c)

Figure 10. Laboratory PQI appliance response to (a) low, (b) intermediate, and (c) high controller gain. The red line is the applied 5-Arms load, and the blue line is the PQI current conduction response. 


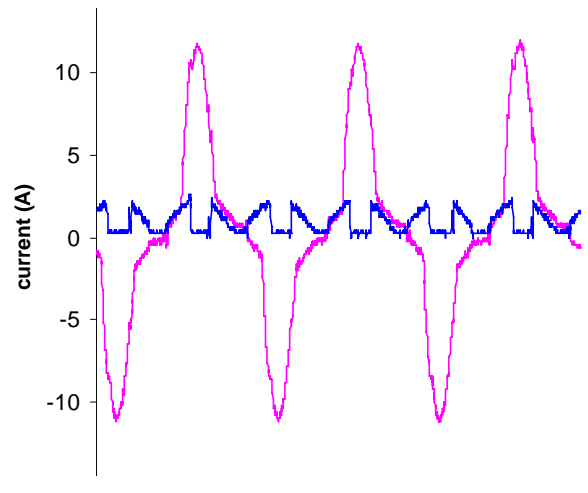

(a)

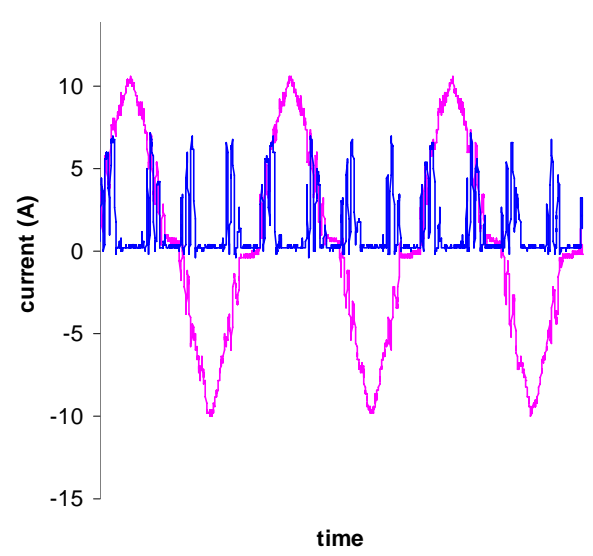

(b)

Figure 11. A change in PQI appliance performance is observed when the impedance of the PQI load is increased from (a) about 120 Ohms to (b) about 24 Ohms. The red current line is that of the electronics load with 5 Arms and

0.8 p.f. The blue line is the current conducted by the PQI appliance.

\section{CONCLUSIONS}

In this paper, an innovative and low cost "power quality improving” appliance technology was introduced to improve aggregate harmonic currents and power factor for building loads. By modulating the current consumption of linear resistive loads, one can make the load preferentially consume harmonic currents and therefore remove much of the unwanted harmonic contents from a building's aggregate load. Furthermore, the power factor of the building will be improved. Unlike conventional electrical power filters, no energy storage devices are needed. The theoretical efficiency of the PQI appliance approaches unity because both the unwanted harmonic components and what would otherwise be electronic conduction and switching losses can be put to use for heating water or other purposes. The initial prototype was inexpensive, suggesting that this approach may lead to inexpensive alternatives to active shunt filters at buildings' point of connection. Both preliminary modeling results and qualitative experimental results suggested that PQI appliances could be a useful tool to improve both the harmonic power quality and power factor within buildings.

\section{REFERENCES}

[1] H. Akagi, "New Trends in Active Filters for Power Conditioning," IEEE Trans. on Industry Applications, vol. 32, no. 6, July/Aug.1996.

[2] S. M. Halpin, "Comparison of IEEE and IEC harmonic Standards," Proc. of Power Engineering Society General Meeting, vol. 3, pp. 22142216, June 2005.

[3] "IEEE Recommended Practices and Requirements for Harmonic Control in Electrical Power Systems", IEEE Std. 519-1992.

[4] B. Singh, V. Verma, A. Chandra, and K. Al-Haddad, "Hybrid filters for power quality improvement," IEE Proc.-Gener. Transm. Distrib., vol. 152, no. 3, pp 365-378, May 2005.

[5] J. Lai and T. S. Key, "Effectiveness of Harmonic Mitigation Equipment for Commercial Office Buildings," IEEE Trans. on Industry Applications, vol. 33, no. 4, July/Aug.1997.

[6] F. J. Peterson, J. E. Patton, M. E. Miller, R. A. Gillman, W. M. Warwick, and W. F. Sandusky, "End-Use Load and Consumer Assessment Program,” Energy and Buildings, ISSN 0378-7788, vol. 19, No. 3, 1993.

[7] O. Garcia, J.A. Cobos, R. Prieto, P. Alou, and J. Uceda, "Single phase power factor correction: a survey," IEEE Transactions on Power Electronics, vol. 18, pp. 749-755, May 2003.

[8] S. Basu and M.H.J. Bollen, "A novel common power factor correction scheme for homes and offices," IEEE Transactions on Power Delivery, vol. 20, pp. 2257-2263, July 2005. 